\title{
FINGER PLETHYSMOGRAPH TO MONITOR HEART RATE
}

\author{
Prachi Samarth $^{1}$, Trinetra V. Naik ${ }^{2}$, S.P. Tondare ${ }^{3}$ \\ ${ }^{1,2}$ Student Departmeant of Electronics (Biomedical)Engineering \\ ${ }^{3}$ Assistant Professor Bharati Vidyapeeth Deemed University College of Engineering, Pune, Maharashtra, India
}

\begin{abstract}
Heart rate is very important physiological parameter that is directly concerned with the soundness of human cardiovascular system. The condition of the cardiovascular system is examined by haemodynamic measurements and by recording the electrical activity of the heart and heart sound. To estimate the performance of the heart as a pump, measurement of cardiac output, blood pressure, blood volume are important and it is carried out in various ways throughout the circulatory system. This paper describes the noninvasive technique to measure the variation in the blood volume in tissue using a pulse sensor which contains a light source and a detector. As the change in blood volume is related to simultaneous occurrence the heart beat, this method can be used to calculate the heart rate.
\end{abstract}

Keywords: Finger Plethysmograph, Heart Rate, Pulse Sensor

\section{INTRODUCTION}

Pulse is usually called as heart rate, which is number of times heart beats each minute (bpm).It gives information about how well the heart is working. It checks general health and overall fitness level. There are various techniques to measure pulse or heart rate. Heart rate is very important parameter in medical field. Average heart rate of human being is adult male is $70 \mathrm{bpm}$ and $75 \mathrm{bpm}$ for adult female, babies heart rate ranges around $120 \mathrm{bpm}$ and older children it is around $90 \mathrm{bpm}$. Heart rate increases during exercise and varies with fitness and age.If heart rate is lower than the threshold level then bradycardia occurs and if higher, then tachycardia. A Plethysmograph is an instrument for recording and measuring the variation in the volume of a part of the body.

There are many costly and sophisticated medical instruments which provide very accurate and precise services to patient regarding diagnosis and treatment point of view. But here we are making an attempt to design low cost heart rate measuring devices using modern technology, which can be used easily by individuals even in rural areas

Plethysmography Method:

Four main type of plethysmograph exist. They include Air displacement, strain gauge, Impedance and photoelectric.

The most commonly used method to measure pulsatile blood volume changes is by the photoelectric method.

In Air displacement plethysmography,the volume of an object is measured indirectly by determining the volume of air it displaces inside an enclosed chamber. Thus, human body volume is measured when a subject sits inside the chamber and displaces a volume of air equal to body. Strain gauge plethysmography uses a transducer filled with mercury or indium-gallium metal alloy conductor. The relative volume change of a limb equals the relative change of the strain gauge wrapped around the limb. Impedance plethysmography based on the measurement of electric impedance at body surface.. The final type is photoelectric plethysmography .In this electrode is used consisting of an infrared LED and a Photo sensor is attached to the skin. The change in volume caused by the pressure pulse is detected by illuminating the skin with the light from LED and then measuring the amount of light either transmitted or reflected to a photo sensor.

\section{Heart Rate Measurement Method:}

Heart rate can be measured by various parameters

-by palpation method is at the wrist (radial artery), the neck (carotid artery), the elbow (brachial artery) and femoral artery. These are manual methods.

-heart rate monitor or ECG is also used for heart rate measurement. This method is known as monitor method.

\section{METHODOLOGY:}

\subsection{System Configuration}

The sensor module of this project consists of a pulse sensor. Front side contains LED which makes contact with the skin. LED glows from the back side and there is a little square just under the LED. The square is a light sensor. The LED radiate light into the fingertip or earlobe, or other capillary tissue, and sensor detect and reads the light that bounces back. The pulse signal from the detector is then get amplified and filtered by op-amp before feeding to microcontroller Atmega 328 for analysis. The microcontroller counts the number of pulses over a fixed time interval and thus obtains the heart rate of the subject. Below is the block diagram of heart rate counter.

Input from the transformer which is $12 \mathrm{~V}$ is connected to the bridge rectifier. Bridge rectifier will convert $\mathrm{AC}$ to $\mathrm{DC}$. Output of bridge rectifier is fed to 1000 microfarad capacitor. Capacitor output is given to regulator. This will 
regulate the transformer output and step down it to $5 \mathrm{~V}$.Next to it one capacitor of 10 microfarad is connected which will remove noise and repulses from the input. Now pulse sensor and power supply circuit is connected to microcontroller

ATmega328. This will take input from sensor and process it for reading. Microcontroller output is given to $16 \times 2 \mathrm{LCD}$ display, which will show heart rate of an person. Along with these circuitry one reset button and buzzer is connected to the microcontroller .If heart rate exceeds above $120 \mathrm{bpm}$ and goes below $20 \mathrm{bpm}$,buzzer will get activated. We are using Arduino software which is open source software that makes it easier for coding and uploading to microcontroller.

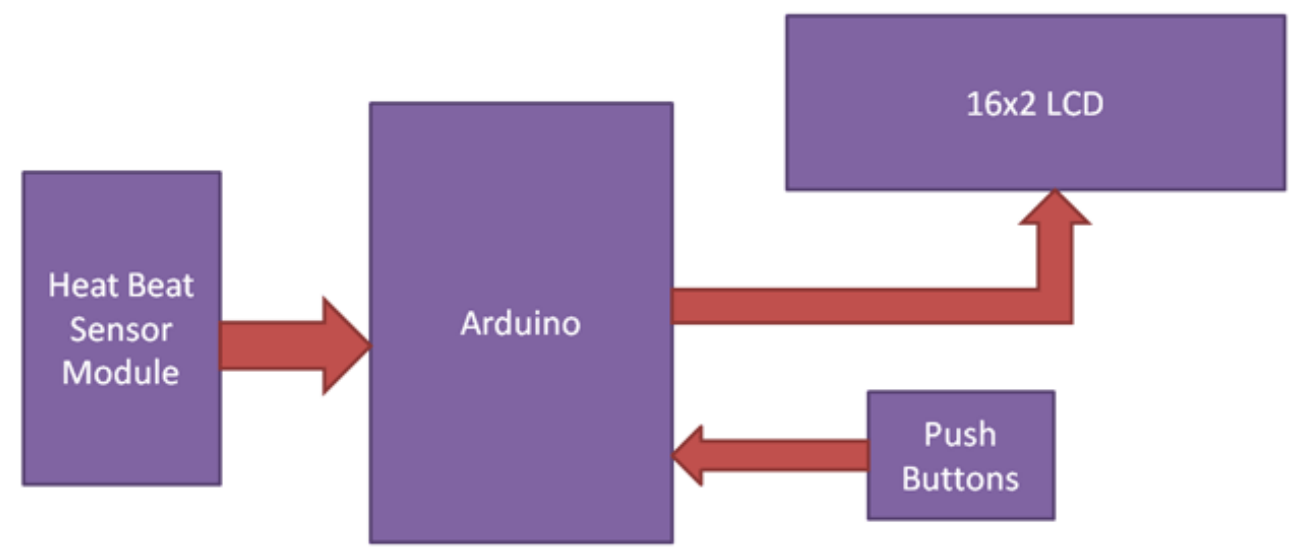

Figure1: Block diagram of "Finger Plethysmograph to monitor Heart rate"

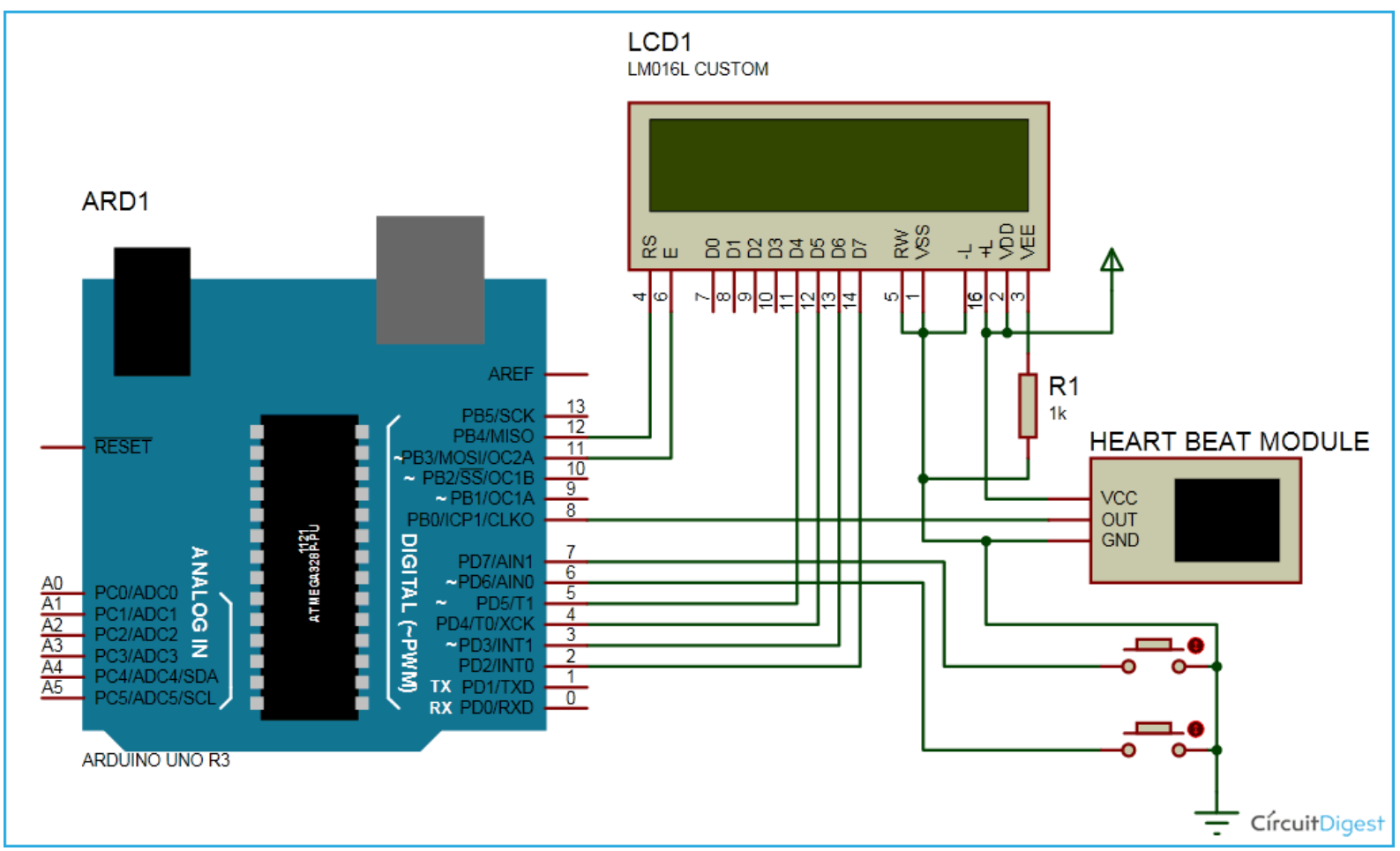

Figure 2: Pin connection of microcontroller with LCD

\section{WORKING WITH SUBJECT}

As per system configuration when power will get on, LCD will display instruction to put finger on sensor module. Subject will have to place a finger on sensor and keep stable for some seconds. Now sensor will collect signal from subject's finger and fed it to microcontroller. The count of heart rate will be displayed on LCD after 15 second. Now for taking next reading subject will have to press reset switch, which will reset the display. And next subject can check their heart rate.

\section{RESULT AND CONCLUSION}

For this project we experimented seven student of range 21 23 years. These students were subject for experiment and study of measurement of heart rate. While switch is on, the display shows instruction for placing fingertip on sensor assembly .After that finger is placed on the sensor. Now subject would wait for 10-15 seconds and should keep calm for some time. After 15 second the result will be displayed. The reset button should be pressed before the next reading is taken. 
From above experiment, we have reached to the conclusion that finger plethysmograph can function as a heart beat detector and works efficiently. It is user friendly. Thus can be used at home .It does not provide any discomfort to the patient. It is portable device. The device can be further improved by adding wireless module to the circuit and other circuitry which can measure multiple physiological parameters in a single system.

Table1: Table shows the recorded data of the subject

\begin{tabular}{|l|l|l|l|}
\hline Subject & Gender & Age & Heart Rate (bpm) \\
\hline Subject1 & Male & 22 & 75 \\
\hline Subject2 & Female & 26 & 82 \\
\hline Subject3 & Male & 21 & 70 \\
\hline Subject4 & Female & 22 & 76 \\
\hline Subject5 & Female & 22 & 74 \\
\hline Subject6 & Male & 22 & 69 \\
\hline Subject7 & Male & 23 & 72 \\
\hline
\end{tabular}

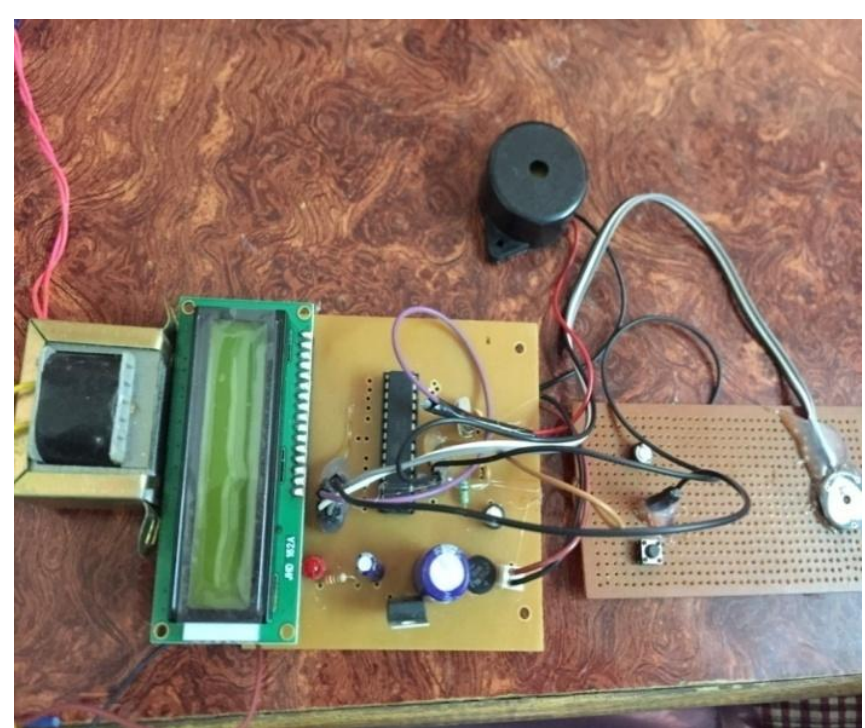

Figure 3: Experimental setup for "Finger Plethysmograph to monitor heart rate"

\section{ACKNOWLEDGEMENT}

We put in our best effort in this project and we are highly obliged to several people for their continuous support.

We are deeply grateful for the regular supervision and guidance of Professor S.P.Tondare and her continuous support throughout the stages of the project The motivation from our family and friends also played an important role in completion of our project.

We would also thank our Principal sir and our college in helping us achieve our desired objective.

\section{REFERANCE}

[1]. Ibrahim D and Buruncuk K.- "Heart Rate Measurement from the Finger Using A Low-Cost Microcontroller"

[2]. Rotariu et al. -"Telemedicine System for Remote Blood Pressure and Heart Rate Monitoring", Proceedings of the 3rd International Conference on E-Health and Bioengineering, 2011

[3]. V. Jayasree, et al., "Design and Development Of a Simple Hardware Setup for Sensing Blood Volume Pulse and a PIC Microcontroller Based Heart Rate Meter," in Biomedical and Pharmaceutical Engineering,2006. ICBPE 2006. International Conference on, 2006, pp. 256-258.

[4]. D. Ibrahim and K. Buruncuk, "Heart Rate Measurement from the Finger Using a Low- Cost Microcontroller," Near East University, Faculty Of Engineering, TRN, 2005.

[5]. Drinnan .M. J, Allen. J and Murray .A. (2001): "Relation between heart rate and pulse transit time during paced respiration", Physiol. Meas. Electronic press.

[6]. Jeong. C. (2006): “A new method to estimate arterial blood pressure using Photo-plethysmographic signal", Platinum press. 